\title{
Cuantificación de vulnerabilidad y riesgo: las inundaciones en Motozintla de Mendoza, Chiapas, México*
}

\author{
Mary Frances Rodríguez Van Gort ${ }^{* *}$
}

Recibido 16 de abril de 2018, aceptado 20 de septiembre de 2018

\section{Resumen}

El objetivo fundamental de esta investigación fue desarrollar una metodología para cuantificar la vulnerabilidad social global. En este estudio la metodología es aplicada para determinar el riesgo por inundación que registra Motozintla. Sin embargo, dicha metodología puede ser replicada en otras comunidades del país y ante otros peligros naturales.

La metodología consistió en (a) realizar la actualización cartográfica del lugar, (b) establecer la muestra mínima de viviendas a caracterizar y seleccionarlas con un método aleatorio simple, (c) determinar las variables fundamentales para la identificación de la vulnerabilidad por grupos (estructural, socio-económica, organizacional, por percepción de riesgo) y global (todas las anteriores por nivel de incidencia ponderado), (d) aplicar el método de interpolación de Distancia Inversa Ponderada (IDW, por sus siglas en inglés) para identificar áreas y niveles de vulnerabilidad y riesgo, y (e) presentar los resultados cartográficamente en mapas generados por un Sistema de Información Geográfica (SIG) para su análisis espacial.

Palabras clave: vulnerabilidad, peligro por inundación, riesgo, Sistemas de Información Geográfica.

\section{Resumo}

O objetivo fundamental desta investigação foi desenvolver uma metodologia para quantificar a vulnerabilidade social global. Neste estudo a metodologia é aplicada

* Ésta es una versión revisada y corregida de una parte del trabajo de tesis doctoral denominada "Cuantificación del riesgo asociado al peligro por inundación en Motozintla de Mendoza, Chiapas, México".

** Profesora de Tiempo Completo del Colegio de Geografía, Facultad de Filosofía y Letras, Universidad Nacional Autónoma de México, Av. Universidad 3000, Col. Ciudad Universitaria, 04510, Alcaldía de Coyoacán, Ciudad de México, correo electrónico: francesrv@gmail.com,cls@filos.unam.mx. 
para determinar o risco por inundação que registra Motozintla. Entretanto, esta metodologia pode ser replicada em outras comunidades do país e diante outros perigos naturais.

A metodologia consistiu em (a) realizar a atualização cartográfica do lugar, (b) estabelecer a amostra mínima de domicílios e selecioná-las com um método aleatório simples, (c) determinar as variáveis fundamentais para a identificação da vulnerabilidade por grupos (estrutural, sócio-econômico, organizacional, por percepção de risco) e global (todas as anteriores por nível de incidência ponderada), (d) aplicar o método de interpolação de Distância Inversa Ponderada (IDW, por sua sigla en inglês) para identificar áreas e níveis de vulnerabilidade e risco, e (e) apresentar os resultados cartograficamente em mapas gerados por um Sistema de Informação Geográfica (SIG) para sua análise espacial.

Palavras chave: vulnerabilidade, perigo por inundação, risco, Sistemas de Informação Geográfica.

\begin{abstract}
The central objective of this research was to develop a methodology for the quantification of global social vulnerability. The methodology is used to determine the risk of flooding in Motozintla. However, such methodology can be replicated in others communities in Mexico.

The methodology consisted in the following steps: (a) the cartographic actualization of the urban layout of Motozintla; (b) the establishment and characterization of a minimal sample of households for this study; (c) selection of variables for the identification of different types of vulnerabilities (structural, socio-economic, organizational, and perception of risk) and global vulnerability (all variables of the analyzed vulnerabilities considering a level of weighted incidence); (d) use of the interpolation method, Inverse Distance Weighting (IDW), to identify areas and levels of vulnerability and risk; and (e) use of a Geographic Information System for cartographic representation of the results and their spatial analysis.
\end{abstract}

Keywords: vulnerability, flood hazard, risk, Geographic Information System.

\title{
Introducción
}

Resulta importante señalar que las comunidades urbanas presentan un número cada vez mayor de desastres en un menor tiempo, lo cual se debe fundamentalmente a la creciente y deficiente urbanización de nuestra sociedad, así como al aumento de exposición ante diversos fenómenos. De acuerdo con los antecedentes presentados por la Organización de las Naciones Unidas (ONU, 2017) en el marco de los Objetivos para el Desarrollo Sostenible, lo anterior puede provocar graves problemas, como el 
crecimiento de asentamientos marginales y la falta de servicios básicos y de infraestructura, lo que aumenta la vulnerabilidad de las comunidades a desastres. Por ello se requiere mejorar la planificación en un sentido de mayor inclusión y gestión urbana que mejore las condiciones de resiliencia y sostenibilidad de los asentamientos.

El propósito fundamental de esta investigación fue desarrollar una metodología para cuantificar la vulnerabilidad social global y a partir de ésta realizar una aproximación al riesgo por inundación en la localidad de Motozintla de Mendoza, Estado de Chiapas, México. Y que este análisis cuantitativo considerara su representación cartográfica a partir de un Sistema de Información Geográfica. Este trabajo forma parte de los resultados de una investigación de doctorado en Ciencias en el área de Geofísica.

La localidad de Motozintla de Mendoza, cabecera municipal Motozintla, se ubica en la porción sureste del estado de Chiapas, entre las coordenadas $15^{\circ} 22^{\prime} \mathrm{N}$ y $92^{\circ}$ $15^{\prime}$ W, con una altitud de 1,260 msnm. De acuerdo al Censo del Instituto Nacional de Estadística, Geografía e Informática (INEGI, 2010a), contaba en 2010 con una población de 23,755 habitantes. Esta comunidad enfrentó en fechas recientes, primero en septiembre de 1998 y luego en octubre de 2005, desastres por inundación y desgajamiento de laderas debido fundamentalmente a las amenazas naturales a que está expuesta, como son las precipitaciones intensas resultado de la influencia de los huracanes procedentes de diversas regiones ciclogenéticas, de acuerdo con el Centro Nacional de Prevención de Desastres (CENAPRED 2001).

El régimen pluviométrico caracteriza a la zona con una moda anual de entre 1.000 y $2.000 \mathrm{~mm}$ con precipitaciones máximas en 24 horas de entre 200 y $400 \mathrm{~mm}$ (Vidal y Gómez, 2007; Vidal, 2007). Son varios los procesos de remoción en masa a los que es propenso su territorio y al combinarse con la vulnerabilidad física y social de sus habitantes se han visto favorecidas las condiciones de susceptibilidad a las inundaciones que pueden derivar en desastres.

La vulnerabilidad global se estimó considerando la vulnerabilidad estructural de las viviendas, la vulnerabilidad socio-económica de sus habitantes, la vulnerabilidad institucional (eficiencia de las autoridades correspondientes en el manejo y gestión del riesgo), y la vulnerabilidad asociada a la percepción del riesgo que la población presenta ante el peligro de inundación. También se incorporaron variables sobre la vulnerabilidad organizacional, tanto de la propia población como la relacionada con la implementación de planes de respuesta institucional.

En este trabajo se establece el nivel de riesgo de Motozintla (un territorio expuesto a inundaciones, particularmente en la zona urbana) considerando su vulnerabilidad y el peligro por inundación. Para ello se establecieron los siguientes objetivos particulares. 
- Desarrollar un marco teórico básico para la cuantificación de la vulnerabilidad.

- Determinar y ponderar las variables de mayor incidencia para el establecimiento de niveles de vulnerabilidad y riesgo.

- Elaborar un instrumento de levantamiento de datos sobre vulnerabilidad en campo.

- Cuantificar los siguientes tipos de vulnerabilidades en Motozintla: estructural, socio-económica, organizacional y global, así como de la percepción del riesgo de la población.

- Determinar el riesgo por inundación en Motozintla de Mendoza a partir de la cuantificación de la vulnerabilidad y la incorporación de la caracterización de la amenaza.

- Interpretar los resultados espacialmente, a partir de su integración en un Sistema de Información Geográfica (SIG).

\section{Cuantificación de la vulnerabilidad}

Se considera al riesgo como la probabilidad de una pérdida, el cual depende de tres elementos: los peligros, la exposición y la vulnerabilidad. "Si cualquiera de estos tres elementos aumenta o disminuye, entonces el riesgo aumenta o disminuye, respectivamente" (Crichton, 1999). En términos generales el riesgo resulta de la combinación del peligro y la vulnerabilidad (Magaña, 2012). Dado lo anterior resulta prioritario la valoración de la vulnerabilidad para la reducción del riesgo (Macías, 1999; Macías y Capra, 2005; Magaña, 2012).

Las condiciones de vulnerabilidad de la población representan una parte fundamental en la ecuación del riesgo y debido a la multivariabilidad que presenta su estudio resulta también de gran complejidad. ¿Qué elementos son fundamentales?, ¿cuáles tienen un mayor peso? Así, uno de los principales retos en la valoración del riesgo resulta el logro de la estimación de una vulnerabilidad social para una población determinada (Adger, 2006; Magaña, 2012). Cabe señalar que uno de los propósitos fundamentales de identificar la vulnerabilidad se encuentra ligado directamente con el aumento de la resiliencia de las comunidades en riesgo a partir del conocimiento de su condición de riesgo y de la gestión participativa del riesgo para la reducción de los desastres.

Es así que la vulnerabilidad ha requerido el desarrollo de diferentes metodologías. Muchos autores han optado por analizar un tipo de vulnerabilidad en especial (social, económica o estructural) para un peligro en particular (Martinelli et al., 2008; Novelo-Casanova y Suárez 2010; Saxena et al., 2013). Por ejemplo, se han llevado a cabo diversos intentos para cuantificar la evaluación de la vulnerabilidad mediante métodos estadísticos. Por ejemplo, Martinelli et al. (2008) desarrollaron un procedimiento para evaluar la vulnerabilidad sísmica de los edificios residenciales en Celano, Italia, por su parte Kaynia et al. (2008) exploraron una nueva metodología para 
estimar la vulnerabilidad a los deslizamientos en la localidad de Lichtenstein, en la cual consideraron una aproximación probabilística simple.

El reto principal para determinar la vulnerabilidad es el desarrollo de herramientas de investigación para una evaluación integrada de la vulnerabilidad (vulnerabilidad global), así como la incorporación de los estudios de gobierno con el propósito de reducir el riesgo y la promoción de estrategias de resiliencia (Adger, 2006), asimismo, este autor sugiere que es importante incorporar la percepción del riesgo, como un elemento central de la vulnerabilidad.

La percepción del riesgo es la forma en que una comunidad determinada percibe el riesgo en función de su experiencia en desastres anteriores o del nivel de exposición a estos peligros. Por ello representa un elemento fundamental en la condición de vulnerabilidad de la población, ya que influye tanto en las acciones de prevención como en las de mitigación (Macías, 1999; Bubeck, 2012). Por otra parte la percepción de riesgo tiene, por lo general un carácter temporal (De Vries, 2007). Para determinar la vulnerabilidad global es necesario tener en cuenta los elementos sociales que construyen esta vulnerabilidad. El análisis de la vulnerabilidad global puede contribuir a la implementación de acciones para la reducción del riesgo (Kappes et al., 2011).

Un elemento fundamental para la metodología de este trabajo se basó en la generación de datos a partir del trabajo de campo, herramienta fundamental de acuerdo con Brid (2009), Jacob et al. (2013) y Banning et al. (2012), tanto en la actualización de la traza urbana y la generación de la cartografía básica, como en la toma de información sobre las condiciones estructurales de las viviendas, las condiciones de los servicios, la preparación ante contingencia y la percepción que la población tiene sobre sus condiciones de riesgo.

La metodología para cuantificar la vulnerabilidad se compuso de siete fases: 1) actualización georreferenciada de la traza urbana de la localidad; 2) selección de variables que condicionan la vulnerabilidad y elaboración del instrumento de encuesta; 3) cálculo de la muestra mínima estadísticamente significativa y selección aleatoria de viviendas; 4) levantamiento de encuestas en campo; 5) creación de bases de datos; 6) análisis de datos y ponderación de variables de acuerdo a su influencia en la vulnerabilidad y 7) análisis espacial de la cuantificación de la vulnerabilidad.

\section{Actualización georreferenciada de la traza urbana de la localidad}

Al visitar la zona de estudio por primera vez (octubre de 2009), se observó que la cartografía con la que contaban las autoridades municipales era obsoleta e incompleta, por lo que se procedió a la actualización de la traza urbana. Para ello, se realizó una primera actualización a partir de las imágenes de satélite de Google y se llevó a cabo una segunda visita de campo en noviembre de 2009, con los mapas obtenidos 
en la red se realizaron los recorridos para el levantamiento actual de las calles, y se rediseñó el mapa base de la localidad de Motozintla de Mendoza. A partir de esta base de datos, se realizó una actualización por medio de la identificación de puntos con aparatos de geo-posicionamiento satelital por Receptores de Sistema Global de Navegación por Satélite (GNSS, por Global Navigation Satellite System en inglés), con lo cual se obtuvo la cartografía georreferenciada de la localidad.

\section{Elección de variables para la encuesta}

En esta fase fue importante considerar que los indicadores no fueran excesivos y guardaran significancia, lo cual es fundamental para lograr valorar cuantitativamente la vulnerabilidad (Magaña, 2012). Se observó que para la toma de datos sobre las características de la población, tanto de sus viviendas, como de los habitantes de las mismas, así como de la percepción de riesgo de la población, resulta necesario el trabajo de campo, para lo cual un instrumento importante es la entrevista a partir del uso de cuestionarios básicos. Para la elaboración del instrumento de encuesta final, se determinaron cuatro tipos de vulnerabilidad: estructural, socio-económica, institucional-organizacional y percepción de riesgo. El instrumento final se estructura por 51 reactivos, con tres tipos de preguntas (Tabla 1).

Tabla 1

Variables y formato que se incluyeron en la encuesta final (agrupados por tema)

\begin{tabular}{ccl}
\hline $\begin{array}{c}\text { Total de } \\
\text { reactivos }\end{array}$ & Formato & \multicolumn{1}{c}{ Tema } \\
\hline 3 & Cerrado & Vulnerabilidad estructural \\
13 & Cerrado & Vulnerabilidad social \\
3 & Semiabierto & Vulnerabilidad económica \\
9 & Semiabierto & Vulnerabilidad organizacional-institucional \\
4 & Cerrado & Vulnerabilidad organizacional-institucional \\
18 & De opinión & Percepción de riesgo \\
1 & Cerrado & Percepción de riesgo \\
\hline
\end{tabular}

Fuente: Elaboración propia a partir de dos ejercicios previos de levantamiento de datos que se realizaron en campo. 


\section{Muestra mínima y selección aleatoria de viviendas}

Aplicando la fórmula (1) para el establecimiento de la muestra mínima (Mendenhall et al., 2003), y el universo de viviendas en la zona de estudio que se determinó en 5,000 viviendas, se obtuvo una muestra mínima de 386. A continuación se describen los elementos del cálculo de la muestra mínima:

$$
\mathrm{n}=\mathrm{Z}^{2}(\mathrm{p}+\mathrm{q}) \mathrm{N} /(\mathrm{N}-1) \mathrm{E}^{2}+\mathrm{Z}^{2}(\mathrm{pq})
$$

Tabla 2

Valor de la muestra mínima para Motozintla de Mendoza a partir de la fórmula 1

\begin{tabular}{cll}
\hline Variable & Descripción de la variable & Valor de la variable \\
\hline $\mathrm{Z}$ & Nivel de confianza & $95 \%$ \\
$\mathrm{p}$ & Variabilidad positiva & .95 \\
$\mathrm{q}$ & Variabilidad negativa & .05 \\
$\mathrm{~N}$ & Tamaño de la población & 5000 \\
$\mathrm{E}$ & Precisión o el error & $5 \%$ \\
$\mathrm{n}$ & Tamaño de la muestra & 386 \\
\hline
\end{tabular}

Fuente: Mendenhall et al., 2003.

En base a estos datos y con la cartografía digitalizada, se realizó una tercera visita de campo a la zona de estudio con la finalidad específica de realizar un conteo de manzanas, lotes y viviendas que sirvieran para la conformación de una base de datos que permitiría realizar, a través del corrimiento de uno de los programas de ArcGIS 3.9 la elección aleatoria simple de los lotes de la localidad y con ello lograr una mayor representatividad de las viviendas que se encuestarían en el trabajo de campo.

A partir del mapa base que se obtuvo para el levantamiento de encuestas, donde se ubicaron 375 manzanas y 5,600 lotes, se aplicó el método de estratos, donde la muestra total es X y la muestra mínima es Y. Se consideraron 40 estratos a partir de su ubicación geográfica, considerando que cada estrato contara con 140 lotes, a cada uno se le asignaron 10 lotes para encuestar al momento de correr el programa de elección aleatoria. Con esta base, la información se incorporó al programa ArcGIS 3.9 que utiliza un método de elección aleatoria simple, donde cada uno de esos 10 lotes tiene la misma probabilidad de ser elegido. 
En el mapa base se consideraron los 40 estratos o áreas geográficas divididos por colores, al interior de cada manzana se aprecia el número que le fue asignado de un total de 375, y los puntos verdes (500) indican los lotes o viviendas que el programa de elección aleatoria simple que se corrió en ArcGIS 3.9 asignó para la realización de las entrevistas (Figura 1).

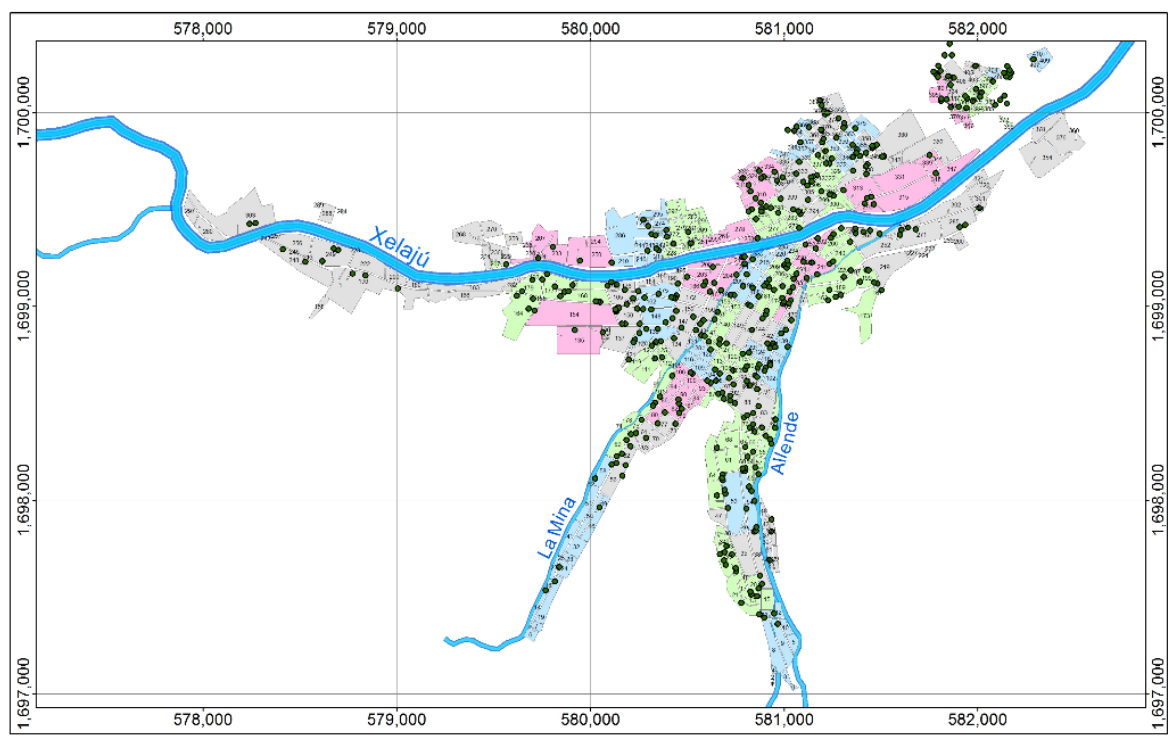

Figura 1. Mapa base de Motozintla de Mendoza (georreferenciado). Los números corresponden a cada una de las 375 manzanas en el área de estudio y los colores a cada uno de los estratos que se determinaron para que la selección aleatoria de viviendas fuera representativa de todo el territorio de la localidad.

\section{Levantamiento de encuestas y creación de la base de datos}

En campo se entrevistaron 486 viviendas, sin embargo, de éstas se descartaron 42 por diversas razones (ilegibilidad, por estar repetidas, o por tener una ubicación errónea, es decir, en vez de vivienda eran lotes baldíos u otro tipo de predio). Por lo tanto, se contó con 444 encuestas finales para la base de datos sobre la vulnerabilidad y la percepción de riesgo. Con estos resultados se creó una planilla de datos en Excel. Posteriormente, se realizaron gráficas (Figura 2) para cada una de las 30 variables seleccionadas para la valoración de la vulnerabilidad (Tabla 3). 

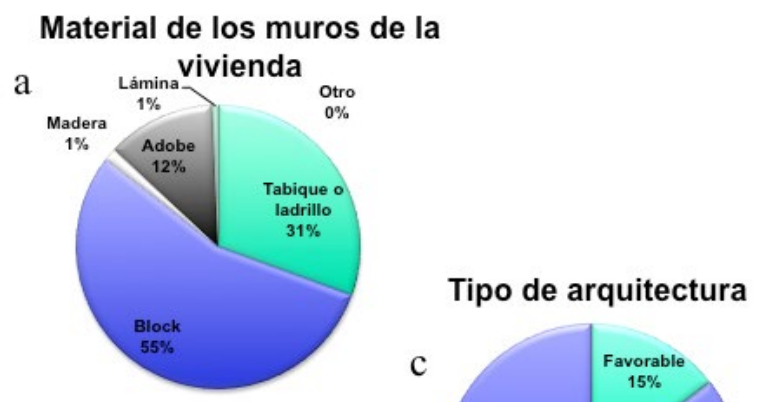

Niveles construidos

$\mathrm{d}$

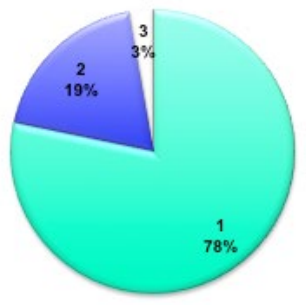

Material del techo de la vivienda

$\mathrm{b}$

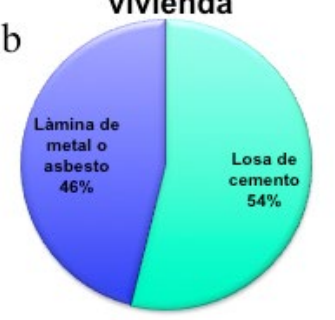

Número de cuartos

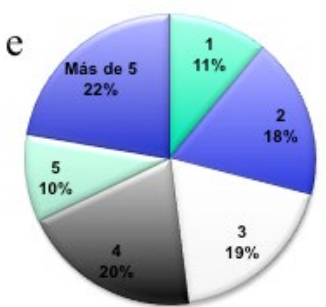

Figura 2. Gráfica de vulnerabilidad estructural, donde se muestran los porcentajes en cuanto a las características estructurales de las viviendas: a) materiales de las paredes, b) materiales del techo, c) tipo de arquitectura, d) niveles de construcción y e) número total de habitaciones en la vivienda.

\section{Selección de variables para la cuantificación de la vulnerabilidad}

A partir del modelo de árbol de decisiones (Quinlan, 1986; Blockeel y De Raedt, 1998), se seleccionaron 25 variables inicialmente, considerando que para la valoración de la vulnerabilidad, cada una de ellas constituye un elemento fundamental que aumenta el nivel de vulnerabilidad. Durante el proceso de análisis de los datos se observó que la ponderación de variables debería incorporar la mayor cantidad de datos que por su calidad de representación permitieran un mejor nivel de precisión en la cuantificación de la vulnerabilidad, por lo que se procedió a una nueva selección, en esta ocasión de 30 variables. A partir de la elección de variables se ponderó el nivel de vulnerabilidad de acuerdo al número de variables presentes para cada tipo de vulnerabilidad así como para la vulnerabilidad global (Tabla 3). 
Tabla 3

Elección de variables y asignación de niveles de vulnerabilidad de acuerdo al número de variables presentes

\begin{tabular}{|c|c|c|}
\hline $\begin{array}{c}\text { Tipo de } \\
\text { vulnerabilidad }\end{array}$ & Variables & $\begin{array}{c}\text { Número de variables } \\
\text { que determinan el nivel } \\
\text { de vulnerabilidad }\end{array}$ \\
\hline Estructural & $\begin{array}{l}\text { Paredes de material distinto de tabique } \\
\text { rojo (generalmente presentan paredes de } \\
\text { block) } \\
\text { Techo de lámina } \\
\text { Un nivel de construcción } \\
\text { Hasta dos habitaciones de construcción } \\
\text { como máximo } \\
\text { Arquitectura desfavorable }\end{array}$ & $\begin{array}{l}\text { Muy bajo: } 1 \\
\text { Bajo: } 2 \\
\text { Moderado: } 3 \\
\text { Alto: } 4 \\
\text { Muy alto: } 5\end{array}$ \\
\hline Socio-económica & $\begin{array}{l}\text { Más de seis habitantes en la vivienda } \\
\text { Algún miembro de la familia mayor de } 70 \\
\text { años } \\
\text { Algún miembro de la familia menor de } 5 \\
\text { años } \\
\text { Algún miembro de la familia analfabeto } \\
\text { Algún miembro de la familia con } \\
\text { discapacidad } \\
\text { Sin servicio médico perteneciente al } \\
\text { IMSS o al ISSSTE } \\
\text { Sin radio ni televisión } \\
\text { Sin teléfono fijo o móvil } \\
\text { Con un ingreso menor a un salario } \\
\text { mínimo por integrante } \\
\text { Sin posibilidad de ahorrar } \\
\text { Sin agua potable } \\
\text { Sin drenaje } \\
\text { Sin energía eléctrica }\end{array}$ & $\begin{array}{l}\text { Muy bajo: } 1 \text { a } 2 \\
\text { Bajo: } 3 \text { a } 4 \\
\text { Moderado: } 5 \text { a } 7 \\
\text { Alto: } 8 \text { a } 9 \\
\text { Muy alto: } 10 \text { o más }\end{array}$ \\
\hline $\begin{array}{l}\text { Organizacional- } \\
\text { institucional }\end{array}$ & $\begin{array}{l}\text { Falta de un Plan de Contingencias } \\
\text { Falta de organización vecinal } \\
\text { Falta de apoyo gubernamental en caso de } \\
\text { inundación } \\
\text { Falta de conocimiento de las rutas de } \\
\text { evacuación } \\
\text { Falta de conocimiento de los refugios } \\
\text { institucionales }\end{array}$ & $\begin{array}{l}\text { Muy bajo: } 1 \\
\text { Bajo: } 2 \\
\text { Moderado: } 3 \\
\text { Alto: } 4 \\
\text { Muy alto: } 5\end{array}$ \\
\hline
\end{tabular}


Continuación Tabla 3

\begin{tabular}{|c|c|c|}
\hline $\begin{array}{c}\text { Tipo de } \\
\text { vulnerabilidad }\end{array}$ & Variables & $\begin{array}{c}\text { Número de variables } \\
\text { que determinan el nivel } \\
\text { de vulnerabilidad }\end{array}$ \\
\hline $\begin{array}{l}\text { Desfavorable } \\
\text { percepción de } \\
\text { riesgo }\end{array}$ & $\begin{array}{l}\text { Los fenómenos naturales suelen } \\
\text { convertirse en desastres } \\
\text { Los desastres no se pueden evitar } \\
\text { Los desastres suelen ser castigo divino } \\
\text { No se manifiesta interesado en los planes } \\
\text { de prevención } \\
\text { No confía en los miembros de su } \\
\text { comunidad } \\
\text { No suele seguir las instrucciones de } \\
\text { Protección Civil } \\
\text { No se manifiesta dispuesto a ser } \\
\text { reubicado a pesar del riesgo al que se } \\
\text { encuentra expuesto }\end{array}$ & $\begin{array}{l}\text { Muy bajo: } 1 \\
\text { Bajo: } 2 \\
\text { Moderado: } 3 \text { a } 5 \\
\text { Alto: } 6 \\
\text { Muy alto: } 7\end{array}$ \\
\hline Global & & $\begin{array}{l}\text { Muy bajo: } 1 \text { a } 6 \\
\text { Bajo: } 7 \text { a } 12 \\
\text { Moderado: } 13 \text { a } 18 \\
\text { Alto: } 19 \text { a } 24 \\
\text { Muy alto: } 25 \text { a } 30\end{array}$ \\
\hline
\end{tabular}

Fuente: Elaboración propia a partir de la ponderación y justificación de variables significativas con base en el trabajo de campo realizado previamente.

\section{Análisis espacial}

A partir de los cuatro grupos de vulnerabilidad analizados (estructural, socio-económica, organizacional-institucional y percepción del riesgo), se llevó a cabo la representación cartográfica de la distribución espacial. Finalmente, se identificó la vulnerabilidad global, considerando la ponderación de la vulnerabilidad en todos los grupos.

A partir de esta identificación de la vulnerabilidad se elaboraron grupos de mapas para cada tipo de vulnerabilidad con diferentes criterios espaciales que permitieran identificar la distribución de la vulnerabilidad en la localidad, tanto por grupo de vulnerabilidades, como por vulnerabilidad global. Los niveles se establecieron a partir de criterios ponderados que permitieran identificar los elementos que son de mayor o menor peso en el aumento de la vulnerabilidad y en el número de variables que se consideraron para el levantamiento de datos. 
Estos mapas se generaron en tres etapas: a) puntuales, por vivienda encuestada; b) por áreas, a partir de la interpolación de datos y c) por manzanas, estableciendo niveles de vulnerabilidad. Se observó que este último análisis espacial resultó el más adecuado para el manejo y gestión del riesgo.

\section{Representación cartográfica puntual (por vivienda encuestada)}

En estos mapas podemos ubicar cada una de las viviendas encuestadas y conocer sus características de vulnerabilidad. Esto nos permite identificar las viviendas con un mayor número de variables desfavorables y "que por lo tanto", presentan un nivel mayor de vulnerabilidad. Cada vivienda encuestada está asociada al punto georreferenciado que se incorporó al momento de la entrevista a partir de un GPS.

Para la valoración de las características de vulnerabilidad estructural, en una primera instancia se consideraron cuatro elementos fundamentales: 1) que sus paredes no fueran de tabique; 2) que el techo fuera de un material distinto de losa; 3) que el número total en la casa fuera de una o dos habitaciones y 4) que solamente tuviera un nivel de construcción. Finalmente se agregó otra variable: 5) que la vivienda presentara una arquitectura desfavorable, es decir, que la altura, el largo y el ancho de los muros la hagan menos resistente. Se consideró que estos elementos son fundamentales para que una vivienda resulte más vulnerable ante un evento de inundación. El mapa muestra que las viviendas que presentan mayor vulnerabilidad se ubican, precisamente al norte del río Xelajú (Figura3).

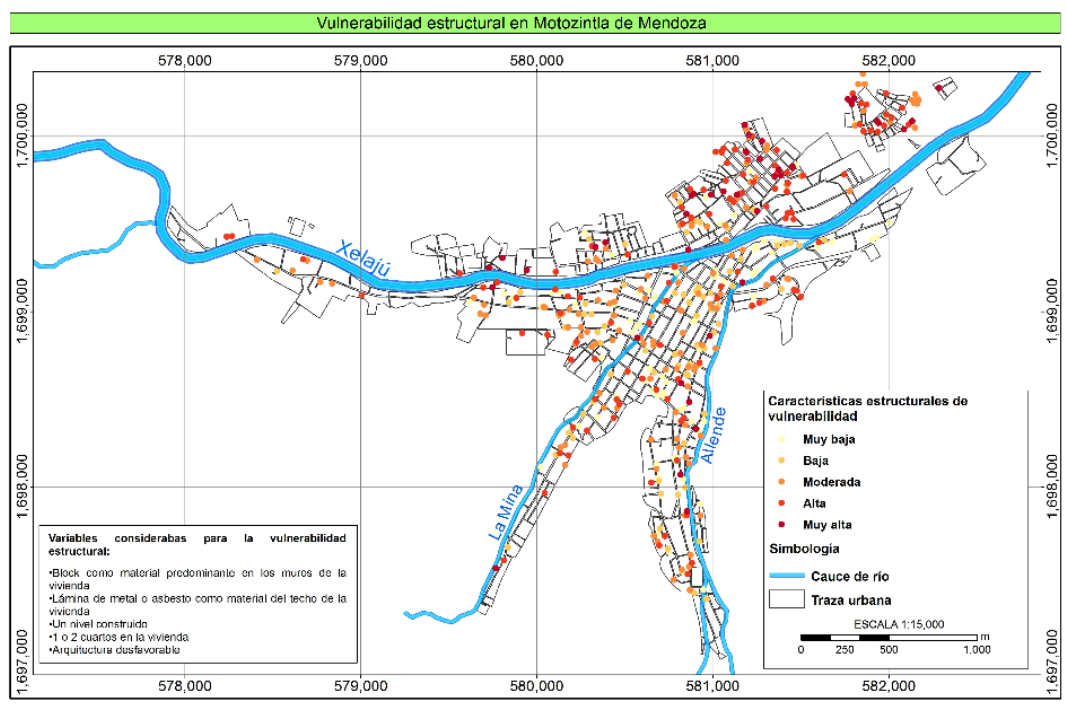

Figura 3. Mapa de viviendas en condiciones de vulnerabilidad estructural en la localidad de Motozintla de Mendoza. 
Representación cartográfica por áreas a partir de la interpolación de datos

El resultado inicial arrojó puntos de mayor y menor vulnerabilidad a partir de la información obtenida sobre las viviendas y las familias levantada en campo, por lo que para identificar áreas que permitieran el análisis espacial de los resultados se utilizó el método de Interpolación de Distancia Inversa Ponderada IDW, con lo que se resaltaron áreas de vulnerabilidad para posteriormente generar una zonificación de riesgo al incorporar las áreas de peligro por inundación. Este método es de gran utilidad en el establecimiento de rangos en las condiciones que se analizan (Lahm, 1983; Watson y Philip, 1985; Philip y Watson, 1986). En un primer ejercicio se utilizó el método de Natural Neighbor, sin embargo, el resultado generó polígonos dispersos dentro del territorio, por lo que se probó con el IDW, que generó resultados de mayor congruencia con los observados en campo.

El análisis de la zonificación de los diversos elementos de la vulnerabilidad indica que las condiciones estructurales de las viviendas presentan mayor vulnerabilidad conforme se aproximan a las periferias, especialmente hacia el norte y noreste de la localidad (Figura 4).

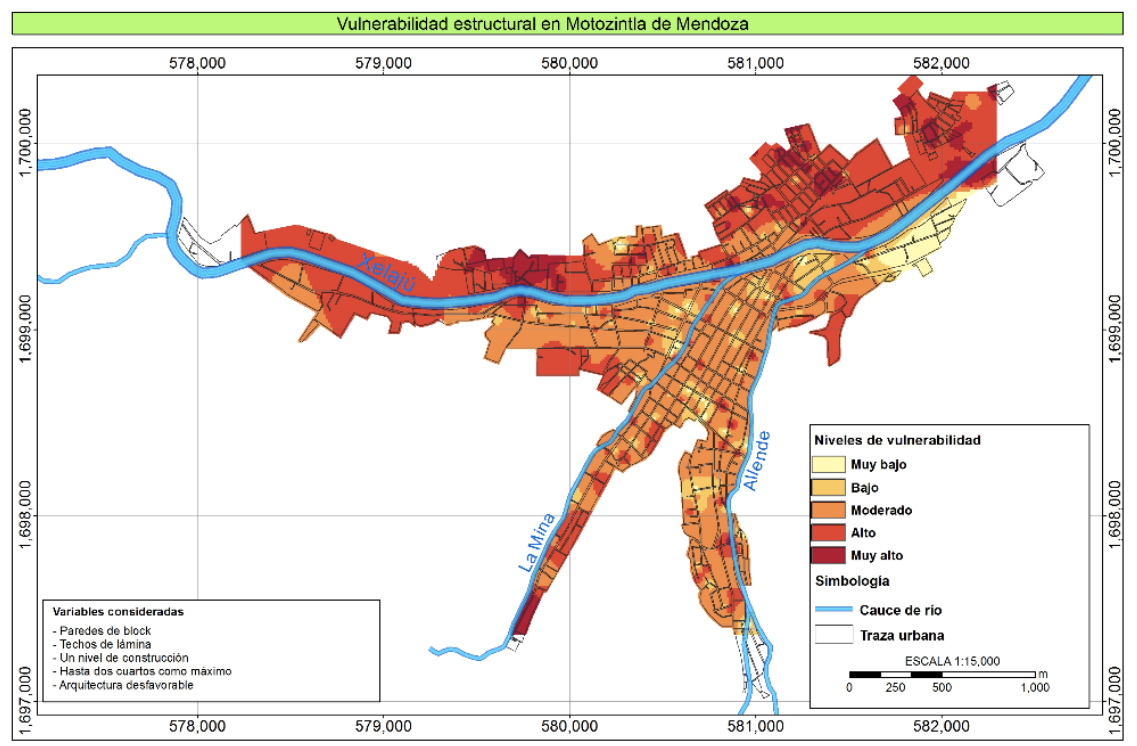

Figura 4. Mapa de vulnerabilidad estructural por áreas interpoladas en Motozintla de Mendoza. 
Al considerar todas las variables seleccionadas para evaluar la vulnerabilidad global (Figura 5), se puede apreciar un patrón similar al caso de la vulnerabilidad estructural, en cuanto al aumento de vulnerabilidad hacia las periferias, sin embargo, se destaca que cuando se incorpora un mayor número de parámetros a considerar, la zonificación resultante es más homogénea en el territorio.

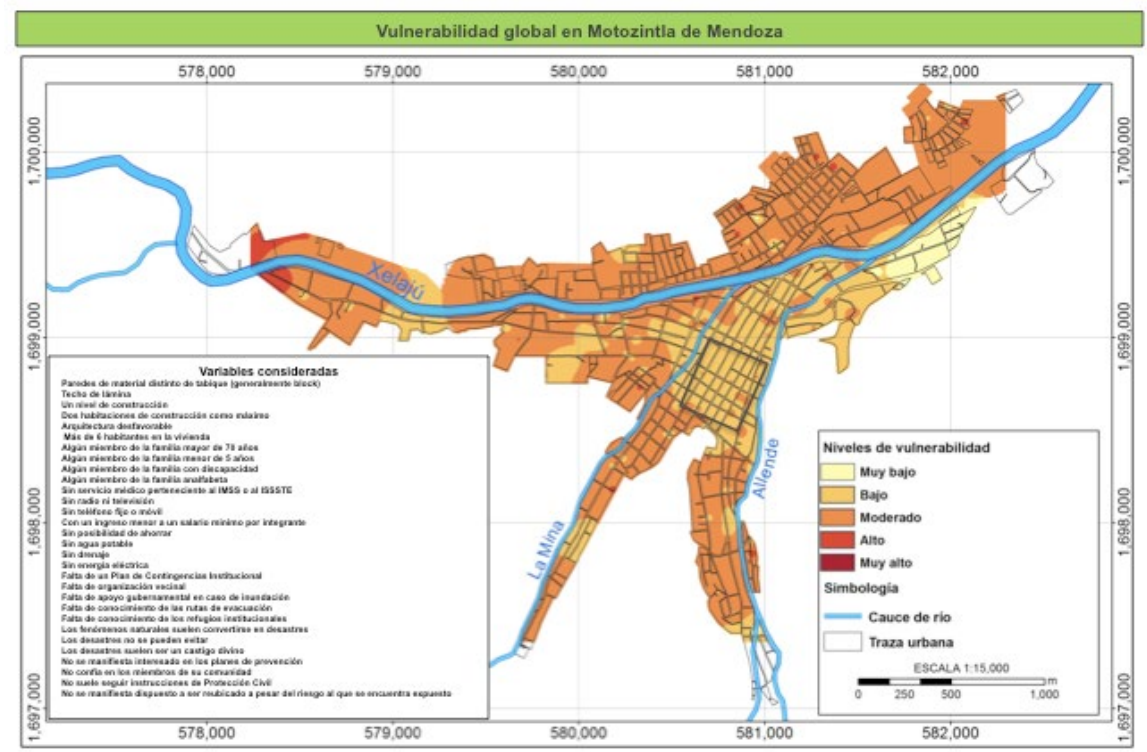

Figura 5. Mapa de vulnerabilidad global por áreas interpoladas en Motozintla de Mendoza.

Representación cartográfica por manzana a partir de la ponderación de niveles de vulnerabilidad

Con el propósito de establecer de manera más precisa la cuantificación de la vulnerabilidad, así como para favorecer el análisis espacial, se optó por incorporar la ponderación de variables de vulnerabilidad por manzana (Tabla 4). Para una mejor representación cartográfica se optó por considerar el promedio de los valores por área interpolada para ajustarlo a la traza urbana. Los resultados que se muestran en estos mapas resultan de mayor precisión, lo cual favorece la gestión de riesgo, tanto para la implementación de medidas de prevención y mitigación, como para la atención de la emergencia. 
Tabla 4

Ponderación de vulnerabilidades dentro de la vulnerabilidad global

$\begin{array}{ccc}\text { Grupo de } & \text { Valor } & \text { Justificación del valor ponderado asignado } \\ \text { vulnerabilidades } & \text { ponderado (\%) } & \end{array}$

Socio-económica

La capacidad económica y social representa el factor determinante en la vulnerabilidad, por lo que se le asignó el mayor peso. Efectivamente, cuando la población no cuenta con servicio médico institucional, se restringe la posibilidad de intervención quirúrgica, por ejemplo. Además si en la familia existe algún integrante en los grupos más vulnerables, por edad, analfabetismo o discapacidad, la vulnerabilidad aumenta. Por otra parte, si no hay suficiente ingreso para solventar la dinámica familiar, en caso de un evento de desastre, la familia queda completamente desamparada y a la espera de apoyo gubernamental, que en el caso de México suele ser deficiente

Estructural

Percepción del riesgo
25

Las características de la vivienda son fundamentales para la resistencia a los fenómenos naturales sin embargo, se consideró de menor influencia con respecto a la vulnerabilidad socio-económica, ya que a su vez la vulnerabilidad estructural, depende del nivel socio-económico de la familia. Así, las viviendas presentan techos de lámina y paredes poco resistentes debido a que la familia cuenta con escasos ingresos. De igual manera influye en el número de habitaciones con los que cuenta la vivienda

La percepción de riesgo impacta en todas las acciones de mitigación y prevención del riesgo de la población. Sin embargo, se consideró una vulnerabilidad menor que la socio-económica y la estructural. Fundamentalmente debido a que la mayoría de la población manifestó una percepción bastante homogénea, donde los fenómenos naturales suelen convertirse en riesgos y éstos difícilmente se pueden prevenir 
Continuación Tabla 4

\begin{tabular}{|c|c|c|}
\hline $\begin{array}{c}\text { Grupo de } \\
\text { vulnerabilidades }\end{array}$ & $\begin{array}{c}\text { Valor } \\
\text { ponderado (\%) }\end{array}$ & Justificación del valor ponderado asignado \\
\hline Institucional & 15 & $\begin{array}{l}\text { Aunque la capacidad institucional es } \\
\text { fundamental para la gestión de riesgo, en esta } \\
\text { investigación se consideró como la de menor } \\
\text { impacto de todas las que se analizaron. Debido } \\
\text { principalmente a que los datos obtenidos en este } \\
\text { rubro no fueron suficientemente exhaustivos para } \\
\text { determinar con claridad su influencia en la } \\
\text { vulnerabilidad. Por ejemplo, se consideró el } \\
\text { conocimiento de la población acerca del plan de } \\
\text { contingencias, a lo que los entrevistados } \\
\text { respondieron que sí lo conocían pero no sabían } \\
\text { en qué consistía }\end{array}$ \\
\hline $\begin{array}{l}\text { Vulnerabilidad } \\
\text { global }\end{array}$ & 100 & $\begin{array}{l}\text { En este caso se consideró la suma de las } \\
\text { vulnerabilidades anteriores }\end{array}$ \\
\hline
\end{tabular}

Fuente: Elaboración propia a partir de la ponderación de variables considerando el nivel de porcentaje de participación o incidencia en la vulnerabilidad global.

En los mapas que resultan de esta ponderación de niveles de vulnerabilidad podemos apreciar con mayor claridad las áreas de vulnerabilidad en el territorio de la localidad. Por ejemplo, el mapa de vulnerabilidad global muestra mayor diferenciación en los niveles de vulnerabilidad y por lo tanto en la identificación más clara de las áreas de mayor y menor vulnerabilidad en la localidad, lo que permite un manejo más adecuado de la información para la gestión de riesgos. La mayor parte del territorio se representa como de media vulnerabilidad en la zona centro y como de alta vulnerabilidad en el resto del territorio (Figura 6).

\section{Peligro por inundación}

Las áreas que presentan susceptibilidad de inundación (Figura 7) se determinaron a partir de la caracterización de la escorrentía en la cuenca de Motozintla realizada por Cantarero (2013), que para realizar la modelación hidráulica utilizó un SIG para integrar las capas en el modelo HEC-RAS y obtener los perfiles hidráulicos a partir de las líneas de corriente central, las orillas del canal principal y las líneas de centro de masa de flujo. Las capas se delinearon a partir de ortofotos aéreas usando el Google 
Earth para su digitalización. Además incorporó una capa con el coeficiente de rugosidad de Manning, para describir la resistencia a la velocidad del cauce por el terreno, a partir del análisis de los usos de suelo. Además del área inundada en el evento del 4 de octubre de 2005 (debido a que considerar los eventos pasados es el primer insumo en la caracterización del peligro).

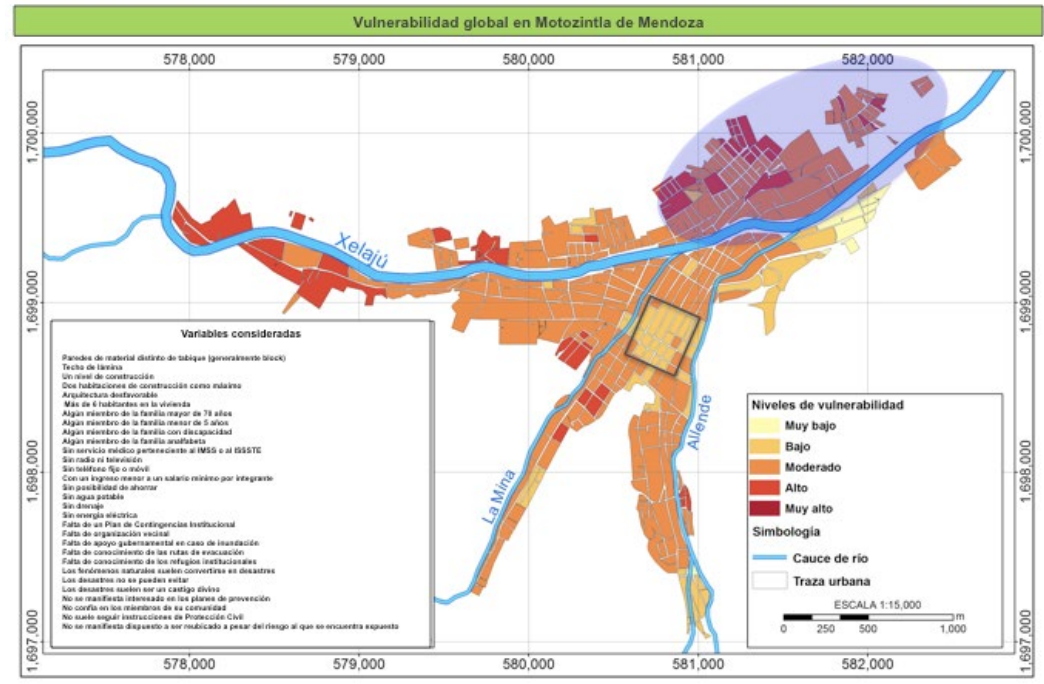

Figura 6. Mapa de vulnerabilidad global.

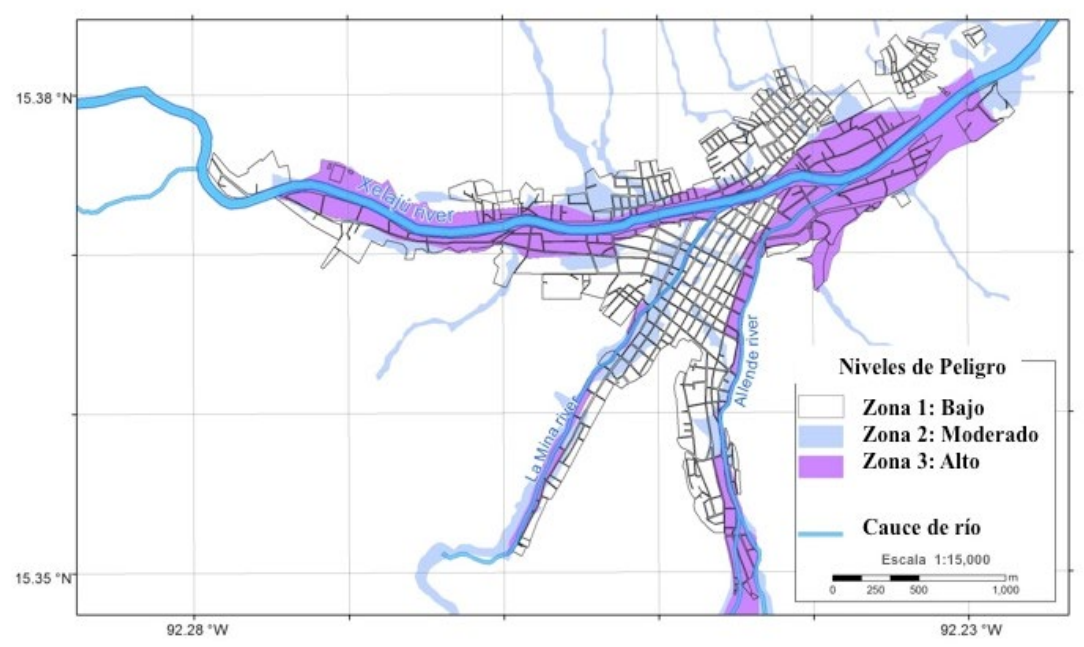

Figura 7. Mapa de susceptibilidad de peligro por inundación en Motozintla de Mendoza. 
Para efectos de esta investigación se consideró únicamente la zona urbana del municipio de Motozintla, la cual corresponde a la cabecera municipal. En este mapa se puede apreciar la zona 1, con peligro bajo, como aquella donde existen asentamientos, pero donde no se tiene registro de que se hayan presentado inundaciones y que tampoco se ubican dentro del área de ninguno de los escenarios de inundación. Se designó como zona 2, peligro moderado al área que representa el escenario menos frecuente, el cual corresponde a un periodo de recurrencia de 20 años (Suárez 2006).

Finalmente, el área inundada el 5 de octubre de 2005 se designó como zona 3, peligro alto, es decir, con la mayor probabilidad de inundación. Para esta asignación se consideró que la precipitación del 4 de octubre registrada en la estación meteorológica El Novillero, localizada a unos $60 \mathrm{~km}$ en línea recta de Motozintla, en la parte alta de la zona, fue de $307 \mathrm{~mm}$ en 24 horas, de acuerdo al Servicio Meteorológico Nacional (SMN) (2005) y el cual según Suárez (2006) en (Cantarero, 2013), corresponde a un periodo de recurrencia de cinco años.

\section{Análisis espacial del riesgo}

La elaboración de matrices y mapas de riesgo se realizó a partir de la identificación de las condiciones de vulnerabilidad, así como de la ponderación de las variables de la vulnerabilidad en las áreas de exposición ante inundaciones. Se consideró como área geográfica básica la manzana y se asignaron valores de vulnerabilidad considerando que los puntos encuestados por manzana representan más del 50\% de las características referidas en cada mapa. Se obtuvieron cinco mapas de riesgo, uno por cada grupo de vulnerabilidades (estructural, socio-económica, organizacional-institucional y por percepción de riesgo) y el mapa final de riesgo, el cual está asociado a la vulnerabilidad global ponderada.

Para la elaboración de la matriz de riesgo (Tabla 5), se identificaron todas las posibilidades de combinación del peligro con la vulnerabilidad que generan las condiciones de riesgo en cada una de las 375 manzanas que componen el territorio de Motozintla de Mendoza. En la matriz se muestran los niveles de vulnerabilidad que se consideraron para los mapas de vulnerabilidad y para las matrices de riesgo por cada grupo de vulnerabilidad analizado.

Como se muestra en la matriz, el índice de riesgo se calculó a partir del producto de $\mathrm{P}$ (nivel de peligro) y V (nivel de vulnerabilidad). Este producto está expresado en la penúltima columna con un número que representa el valor ponderado del riesgo y finalmente se expresa también en forma cualitativa en la última columna.

Se construyeron mapas de riesgo por cada tipo de vulnerabilidad: estructural, socio-económica, organizacional-institucional y por inadecuada percepción de riesgo. Finalmente se elaboró el mapa de riesgo por inundación asociado a vulnerabilidad global (Figura 8). 
Tabla 5

Matriz de riesgo

\begin{tabular}{|c|c|c|c|}
\hline $\begin{array}{c}\text { RIESGO } \\
\text { Justificación de la escala }\end{array}$ & $\begin{array}{l}\text { Índice } \\
\text { de } \\
\text { riesgo }\end{array}$ & $\begin{array}{c}\text { Valor } \\
\text { ponderado } \\
\text { de riesgo }\end{array}$ & $\begin{array}{l}\text { Nivel de } \\
\text { riesgo }\end{array}$ \\
\hline $\begin{array}{l}\text { Zona sin inundación y sin elementos de } \\
\text { vulnerabilidad }\end{array}$ & 0 & 0 & Sin riesgo \\
\hline Zona sin inundación y vulnerabilidad $1(1 \mathrm{x} 1=1)$ & 1 & 1 & Muy bajo \\
\hline $\begin{array}{l}\text { Zona de inundación potencial y vulnerabilidad } \\
1(2 \times 1=2) \text { Zona sin inundación y vulnerabilidad } \\
2(1 \times 2=2)\end{array}$ & 2 & 2 & Bajo \\
\hline $\begin{array}{l}\text { Zona sin inundación y vulnerabilidad } 3(1 \times 3=3) \\
\text { Zona de inundación potencial y vulnerabilidad } \\
2(2 \times 2=4)\end{array}$ & 3 a 5 & 3 & Moderado \\
\hline $\begin{array}{l}\text { Zona de inundación pasada y vulnerabilidad } 2 \\
(3 \times 2=6) \text { Zona de inundación potencial y } \\
\text { vulnerabilidad } 5(2 \times 5=10) \text { Zona de inundación } \\
\text { pasada y vulnerabilidad } 3(3 \times 3=9)\end{array}$ & 6 a 10 & 4 & Alto \\
\hline $\begin{array}{l}\text { Zona de inundación pasada y vulnerabilidad } 5 \\
(3 \times 5=15)\end{array}$ & 11 a 15 & 5 & Muy alto \\
\hline
\end{tabular}

Fuente: Elaboración propia a partir del establecimiento del índice de riesgo, considerando el peligro por inundación y el nivel de vulnerabilidad.

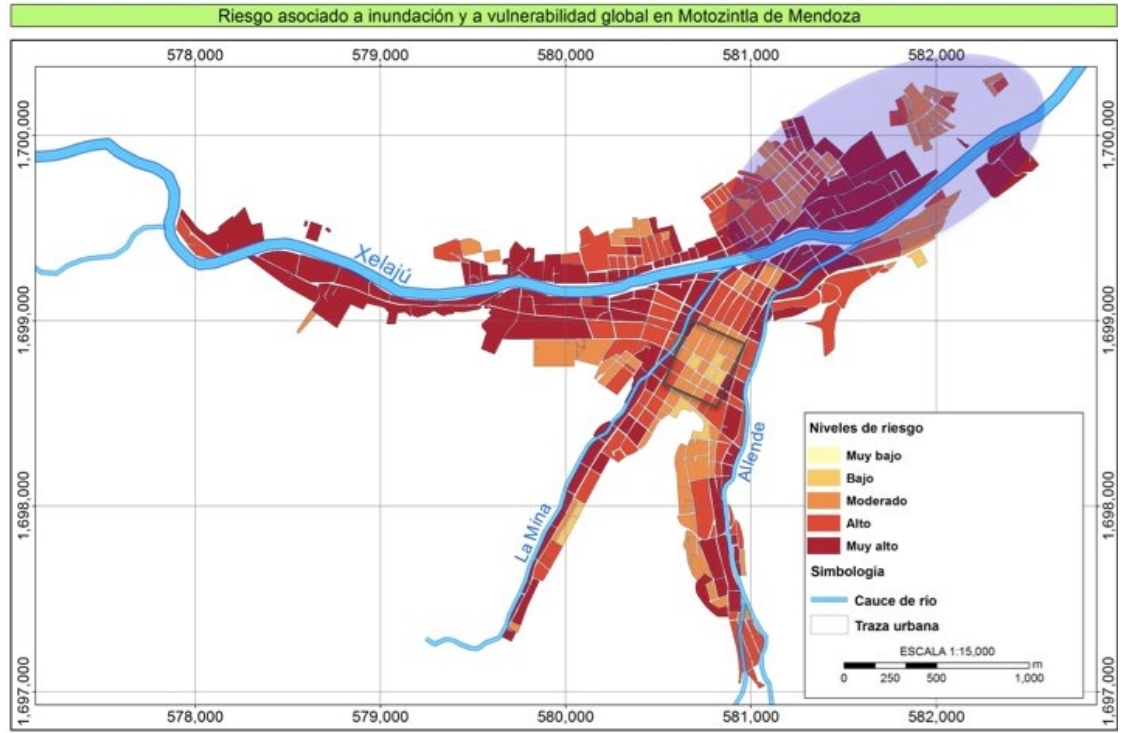

Figura 8. Mapa de riesgo por inundación en Motozintla de Mendoza. 


\section{Conclusiones}

En este trabajo la valoración de la vulnerabilidad en Motozintla quedó referida a aquellos elementos característicos que generan que la población sea en mayor o menor medida susceptible a sufrir daños en su integridad física, sus bienes y su entorno ante la presencia de un evento de desastre, así como que le dificulten o favorezcan su recuperación. Por lo tanto, la vulnerabilidad global se entiende como la serie de variables que nos permiten realizar una cuantificación de la vulnerabilidad a partir de la identificación de las condiciones estructurales de la vivienda, el acceso a los servicios públicos y domiciliarios, las características socioeconómicas, la preparación ante el riesgo de desastre y la percepción de riesgo de la población. Se establece como un hallazgo el hecho de que la elección ponderada de las 30 variables consideradas fuese adecuada para lograr la diferenciación de áreas con distintos niveles de vulnerabilidad al interior de la localidad. De Vries (2011) encontró que la valoración de la vulnerabilidad global es fundamental y Puente (2009) que la ponderación de las variables que inciden en mayor o menor medida es todavía de mayor relevancia.

Los resultados indican que en cuanto a la vulnerabilidad global (Figura 6), se puede observar que aproximadamente un $60 \%$ del territorio de la localidad se ubica en el nivel alto de vulnerabilidad y un $30 \%$ en el intervalo de vulnerabilidad media. Especialmente la porción central y algunas zonas al NE presentan muy alta vulnerabilidad. En general, estas zonas corresponden a los asentamientos reubicados que el gobierno local llevó a cabo después de los eventos de desastre de 1998 y 2005.

En este sentido, se encontró que la vulnerabilidad estructural representa uno de los factores de mayor influencia en la generación de riesgo dentro de la comunidad, por ejemplo, las viviendas en general presentan la mayoría de las variables de vulnerabilidad, ya que predomina el techo de lámina, las viviendas con paredes poco resistentes y la falta de servicios. Se pudo apreciar que el nivel de riesgo que se alcanza en la mayoría del territorio con peligro de inundación es "muy alto". Este resultado indica que en caso de una inundación, las probabilidades de que se pierda total o parcialmente la vivienda son "altas" y "muy altas", respectivamente (Figura 4). Puente (2009) encontró que en la Ciudad de México la vulnerabilidad estructural era significativa para valorar el riesgo sísmico y dentro de los elementos que encontró como relevantes está la construcción con arquitectura desfavorable.

Al ponderar las variables de vulnerabilidad de acuerdo con su incidencia en ésta, se obtuvo una idea más clara sobre el riesgo de Motozintla ante la exposición de inundación, por lo que representa una aportación para la identificación y valoración de riesgo asociado a inundaciones, dentro de los Objetivos de la ONU para el Desarrollo Sostenible. En este sentido el Objetivo "Ciudades y Comunidades Sostenibles", establece siete metas dentro de las cuales se encuentran el asegurar a toda la población de viviendas y servicios básicos adecuados, prestar atención especial a los grupos vulnerables, como son mujeres, niños, personas con discapacidad y adultos 
mayores. Y en el marco de Sendai para la Reducción de los Desastres 2015-2030, reducir significativamente el número de muertes y daños por desastres asociados a fenómenos naturales y aumentar la gestión de riesgos de desastres en todos los niveles de acción.

En cuanto a las ventajas de la metodología presentada en esta investigación para cuantificar la vulnerabilidad y el riesgo, es factible afirmar que puede utilizarse para cualquier otro tipo de peligro o amenaza, adecuando la ponderación de variables de acuerdo con las características del nivel de peligro y de exposición. La unidad espacial seleccionada (la manzana) resultó adecuada ya que permitió manejar un nivel de acercamiento significativo en la identificación del nivel de riesgo y por otra parte resulta óptimo para la gestión del riesgo local.

La toma de datos en campo fue fundamental, ya que por una parte favoreció la corroboración de datos sobre las características estructurales de las viviendas y socioeconómicas de sus habitantes y por la otra permitió la obtención de nuevos datos relacionados con la condición de la población en cuanto a estas características. La elaboración del mapa de la localidad, con la traza urbana actualizada y georreferenciada resultó la base fundamental para el análisis espacial de las condiciones de vulnerabilidad, peligro y riesgo.

El trabajo de campo también permitió obtener datos sobre las condiciones organizacionales, tanto de los habitantes, como de sus autoridades relacionadas con la prevención y la mitigación del impacto de los desastres, así como de la percepción de riesgo.

La elaboración de matrices de vulnerabilidad ponderada permitió establecer áreas de riesgo con mayor nivel de diferenciación. Finalmente, el análisis y representación de la información mediante un SIG permitió establecer niveles espaciales del riesgo dentro de la localidad. Lo cual puede favorecer la gestión integral del riesgo por parte de las autoridades locales.

Los mapas de vulnerabilidad y riesgo generados en la investigación son insumos necesarios para la gestión integral del riesgo en Motozintla de Mendoza. Esta comunidad ahora cuenta con un mapa de la traza urbana actualizado y georreferenciado, así como con mapas de vulnerabilidad diferenciada y global y mapas de riesgo asociado a estas vulnerabilidades y al peligro por inundación. Lo anterior representa una innovación y una aportación para la valoración de riesgo local en general y para Motozintla de Mendoza en particular.

Los mapas de riesgo, al considerar el peligro por inundación y la vulnerabilidad de la población, representan las bases para una adecuada gestión de riesgo, ya sea al calcular costos de las posibles pérdidas, tanto en vivienda como en infraestructura, así como en servicios y productividad, o ya sea para la atención de las emergencias, ya que aportan información valiosa para la toma de decisiones en cuanto a la evacuación y la atención prioritaria (Gobierno de España, 2013). 
Considerando que la valoración del riesgo incluye cierto grado de complejidad en el reconocimiento espacial de los elementos de la vulnerabilidad especialmente para los tomadores de decisiones, se pretende que este trabajo represente una aportación metodológica para la valoración de la vulnerabilidad y el riesgo y su consecuente modificación en el patrón de gestión de riesgos, dentro de lo que cabría promover el desarrollo e implementación de medidas como: Programa de Desarrollo Urbano; Programa de Reordenamiento Ecológico Territorial: Programa de Alerta Temprana Programa de Reducción de Riesgo; Priorización de áreas de atención en caso de contingencia o desastre; Restricción de zonas no aptas para la construcción de viviendas; Programa de Conservación Ambiental; Normas locales de cuidado y preservación ambiental; Iniciativa de Leyes y Reglamentos Municipales y el Fomento y apoyo a iniciativas de acciones comunitarias o vecinales entro otras.

\section{Bibliografía}

Adger, N. (2006). "Vulnerability", Global Environmental Change, 16(3): 268-281. Banning, R.; Camstra, A. and Knottenerus, P. (2012). Sampling Theory: Sampling Design and Estimation Methods, The Hague/Heerlen: Statistics Netherlands.

Bird, D.K. (2009). "The Use of Questionnaires for Acquiring Information on Public Perception of Natural Hazards and Risk Mitigation: A Review of Current Knowledge and Practice", Natural Hazards and Earth System Sciences, 9(4): 1307-1325.

Blockeel, H. and Luc De Raedt, L. (1998). "Top-Down Induction of Logical Decision Trees”, Artificial Intelligence, 101(1-2): 285-297.

Bubeck P.; Botzen, W. and, Aerts, J. (2012). "A Review of Risk Perceptions and other Factors that Influence Flood Mitigation Behavior", Risk Analysis, 32(9): 1481-1495.

Cantarero, F. (2013). "III.7 Zonificación de la Amenaza por Inundaciones", en Atlas de Factores de Riesgos de la Cuenca de Motozintla, Chiapas, coordinado por Oropeza, Oralia y Figueroa, M., Instituto de Geofísica, UNAM, 133-139.

Centro Nacional de Prevención de Desastres (CENAPRED) (2001). Diagnóstico de Peligros e Identificación de Riesgos de Desastres en México, México, Secretaría de Gobernación.

Crichton, D. (1999). "The Risk Triangle." in Natural Disaster Management, edited by Ingleton J. London, Tudor Rose., 102-103.

De Vries, D. (2011). "Temporal Vulnerability in Hazardscapes: Flood Memory-Networks and Referentiality along the North Carolina Neuse River (USA)", Global Environmental Change, 21(1): 154-164.

Instituto Nacional de Estadística, Geografía e Informática (INEGI) (2010a). Censo de Población $\quad y \quad$ Vivienda, disponible en 
$<$ http://www.inegi.org.mx/est/contenidos/proyectos/ccpv/cpv2010/>, consultado el 09 de mayo de 2012).

Jacob, S.; Weeks, P.; Blount, B. and Jepson, M. (2013). "Development and Evaluation of Social Indicators of Vulnerability and Resiliency for Fishing Communities in the Gulf of Mexico", Marine Policy, 37: 86-95.

Kappes, M.; Papathoma-Köhle, M. and Keiler, M. (2011). “Assessing Physical Vulnerability for Multi-Hazards Using an Indicator-based Methodology", Applied Geography 32(2): 577-590.

Kaynia, A.; Papathoma-Köhle, M.; Neuhäuser, B.; Ratzinger, K.; Wenzel, H. and Medina-Cetina, Z. (2008). "Probabilistic Assessment of Vulnerability to Landslide: Application to the Village of Lichtenstein, Baden-Württemberg, Germany" Engineering Geology, 101(1-2): 33-48.

Macías-Medrano, J.M. (1999). Desastres y Protección Civil: Problemas Sociales, Políticos y Organizacionales, Centro de Investigación y Estudios Superiores en Antropología Social (CIESAS), 101 pp.

Macías-Vázquez J.L. y Capra, L. (2005). Los volcanes y sus peligros: Situación actual en México y Latinoamérica, Fondo de Cultura Económica, México, 153 pp.

Magaña, V. (responsable) (2012). Guía Metodológica para la Evaluación de la Vulnerabilidad ante Cambio Climático, Instituto Nacional de Ecología y Cambio Climático (INECC), Programa de las Naciones Unidas para el Desarrollo (PNUD), México, $61 \mathrm{pp}$.

Martinelli, A.; Cifani, G.; Cialone, G.; Corazza, L.; Petracca, A. and Petrucci, G. (2008). "Building Vulnerability Assessment and Damage Scenarios in Celano (Italy) Using a Quick Survey Data-based Methodology", Soil Dynamics and Earthquake Engineering, 28(10-11): 875-889.

Mendenhall, W.; Beaver, R.J. and Beaver, M.B. (2012). "Introduction to Probability and Statistics", Duxbury Press, 744 pp.

Ministerio de Agricultura, Alimentación y Medio Ambiente (2013). Propuesta de mínimos para la metodología de realización de los mapas de riesgo de inundación. Gobierno de España, disponible en $<$ http://www.mapama.gob.es/es/agua/temas/gestion-de-los-riesgos-deinundacion/Metodologia\%20mapas\%20de\%20riesgo\%20Dir\%20Inundaciones\%20JULIO\%202013_tcm30-98530.pdf>, Fecha de consulta: 22/06/2018).

Novelo-Casanova, D. and Suárez, G. (2010). "Natural and Man-made Hazards in the Cayman Islands." Natural Hazards 55: 441-466.

Philip, G.M. and Watson, D. F. (1986). “Automatic Interpolation Methods for Mapping Piezometric Surfaces", Automatica, 22(6): 753-756.

Puente, S. (2010). "Una megalópolis en riesgo: La Ciudad de México y el desafío de la prevención de un riesgo anunciado", en Los grandes problemas de México, Tomo IV Medio Ambiente, coordinado por Lezama, J.L. y Graizbord, B. Centro 
de Estudios Demográficos, Urbanos y Ambientales del Colegio de México, México, 373-397.

Quinlan, J. (1986). "Induction of Decision Trees", Machine Learning 1(1): 81-106.

Saxena, S.; Geethalakshmi, V. and Lakshmanan A. (2013). "Development of habitation vulnerability assessment framework for coastal hazards: Cuddalore coast in Tamil Nadu, India - A case Study" Weather and Climate Extremes (2): 48-57.

Servicio Meteorológico Nacional (SMN) (2005). "Precipitación de 04 de octubre de 2005, El Novillero, Chiapas", disponible en $<$ http://smn.cna.gob.mx/index.php?opcion=com>, consultado el 10 de julio de 2012.

Siu-Ngan Lam, N. (1983). "Spatial Interpolation Methods: A Review", The American Cartographer, 10(2): 129-150.

Suárez, J. (2006). Análisis de los problemas de erosión y deslaves en la carretera Hixtla-Motozintla, Chiapas, Instituto de Erosión y Deslizamientos, Bucaramanga. Colombia, $34 \mathrm{pp}$.

Vidal, R. (2007). "Carta NA IV 11 Precipitación máxima en 24 horas en milímetros", Atlas Nacional, Instituto de Geografia, UNAM, México.

Vidal, R. y Gómez, R.G. (2007). "Carta NA IV 5 Precipitación Total Anual”, Atlas Nacional Instituto de Geografia, UNAM, México.

Watson, D. and Philip, G. (1985). "A Refinement of Inverse Distance Weighted Interpolation”, Geo-Processing, 2(4): 315-327. 